\title{
latrogenic cannula-associated ocular injuries during anterior segment surgery: time to re-think luer-lock design?
}

\author{
Craig Wilde $^{1} \cdot$ Andrew R. Ross $^{1} \cdot$ Gavin Orr ${ }^{1} \cdot$ Harminder Dua $\mathbb{D}^{1}$ \\ Received: 21 August 2018 / Accepted: 7 September 2018 / Published online: 25 October 2018 \\ (c) The Royal College of Ophthalmologists 2018
}

Cataract surgeons are aware of potential iatrogenic ocular injuries from accidental cannula release during injections of solutions or ophthalmic viscosurgical device (OVD) [1, 2]. Prevention advice is simple. Surgeons and theatre nurse practitioners should be aware of the complication, taught to check instrument security and comply with manufacturers user instructions. Surgeons' should firmly hold the cannula base. Universal adoption of luer-lock syringes has been established. Despite these measures however, events still occur.

Although rare (with possible under reporting), incidence during cataract surgery is between 0.009 [1] and $0.07 \%$ [2]. Serious ocular injuries can occur with visual loss being reported particularly with retinal damage [2]. Despite efforts to increase awareness, events still occur. It appears the standard advice to check for tight adherence and hold the cannula hub during injection is not enough to adequately mitigate risk. Even apparently tight fitting cannulas have been reported to dislodge [3], with reports of both luer-lock adapter and cannula becoming detached from the syringe, acting as a projectile [4]. It is still possible that surgeon and theatre nurse both fail to screw and tighten the luer-lock connection or cross threading could occur.

The author $(\mathrm{CW})$ has first-hand experience of being handed an OVD with the luer-lock being accidentally removed by the theatre nurse. Instead of the 'tip cap' of an Amvisc (Bausch and Lomb, Kingston Upon Thames, UK) being screwed off, the entire luer-lock system was removed (by a pulling motion) and cannula affixed, contrary to the manufacturer's instructions (Fig. 1). A twisting/tightening motion can be performed by the surgeon, but unless direct visual inspection of the device is performed, it will not be immediately evident that the luer-lock has been removed. This poses a potential risk and cataract surgeons must be aware that inadvertent detachment of luer-locks is possible (depending on the individual product). It is the authors' opinion that device design should change making inadvertent removal of luer-locks impossible. If iatrogenic cannula injuries are to be completely eradicated from practice, closer liaison with instrument manufacturers is required. Development of integrated syringe-cannula systems to eliminate all possibilities of inadequately tightening or inappropriately removing luer-locks is needed. Simple statements that luer-locks never fail will not avoid future harm if human performance shortfalls cannot be eliminated from every possible step. The safest way to do this is at the manufacturing stage by moulding one-piece cannula-syringe devices, where syringe and cannula hub are moulded together eliminating the possibility of detachment by force or inadequate tightening.

Acknowledgements Photographs taken by Emily Hogan, Queen's Medical Center, Nottingham.

\section{Compliance with ethical standards}

Conflict of interest The authors declare that they have no conflict of interest.
Harminder Dua

harminder.dua@nottingham.ac.uk

1 Ophthalmology and Visual Sciences, Division of Clinical Neurosciences, B Floor, EENT Centre, Queen's Medical Centre, University of Nottingham, Nottingham, UK 

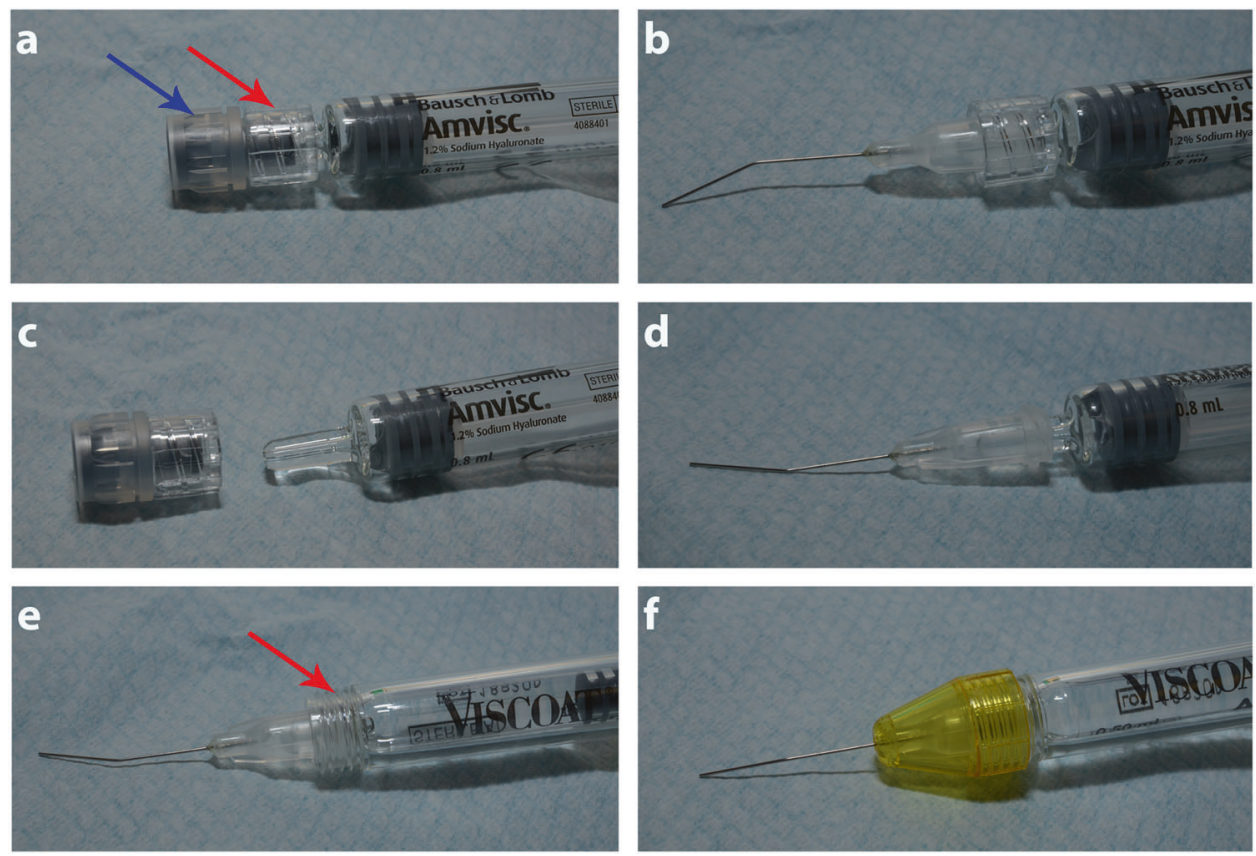

Fig. 1 Amvisc syringe assembly as provided out of packaging (a). The grey 'tip cap' (blue arrow) has to be screwed off the 'luer-lock' (red arrow) and then cannula (with luer) tightened within the luer-lock for correct assembly (b). It is possible to detach the luer-lock from the end of the Amvisc syringe, obviously going against the manufacturers instructions (c). However, this makes it possible for a surgeon to be handed an inappropriately assembled OVD that poses a danger of

forceful detachment (d). An Alcon Viscoat syringe assembly (e). The syringe tip has a non-detachable moulded luer-lock (red arrow) that cannot be removed. To act as a safeguard from failure to correctly tighten the lure-lock by rotating the cannula to the full length of the sleeve threads, the design is improved by the inclusion of a yellow cannula locking cap (f). By applying the locking cap against the cannula hub, the cannula cannot act as a projectile

\section{References}

1. Ting DS, Vaideanu-Collins D, Ellerton C. Cannula-associated ocular injuries during cataract surgery: the North East England Study. Eye. 2016;30:764-5.

2. Rumelt S, Kassif Y, Koropov M, Landa E, Marzuk F, Segal ZI, et al. The spectrum of iatrogenic intraocular injuries caused by inadvertent cannula release during anterior segment surgery. Arch Ophthalmol. 2007;125:889-92.

3. Dinakaran S, Kayarkar VV. Intraoperative ocular damage caused by a cannula. J Cataract Refract Surg. 1999;25:720-1.

4. Verbrugghe $\mathrm{A}$, Hesta $\mathrm{M}$, Gommeren $\mathrm{K}$, Daminet $\mathrm{S}$, Wuyts B, Buyse J, et al. Oligofructose and inulin modulate glucose and amino acid metabolism through propionate production in normal-weight and obese cats. Br J Nutr. 2009; 102:694-702. 